\title{
Primary Sjögren's Syndrome: A Retrospective Cohort Study of Burden of Illness in Sweden
}

\author{
Anna Westerlund · Anne Mette Tranberg Kejs · Heval Beydogan • \\ Kerry Gairy (D)
}

Received: December 22, 2020 / Accepted: April 22, 2021 / Published online: June 9, 2021

(C) The Author(s) 2021

\begin{abstract}
Introduction: Primary Sjögren's syndrome (pSS) is an autoimmune disease that affects salivary and lachrymal glands and is associated with complex extraglandular manifestations. This study investigates the clinical and economic burden and disease course of pSS in Sweden.

Methods: This retrospective cohort study utilizes data from Swedish national registries and consists of patients at least 18 years of age diagnosed in secondary care with pSS, and matched members of the Swedish general population. Healthcare resource utilization (HRU) and costs were compared. The clinical burden of
\end{abstract}

Supplementary Information The online version contains supplementary material available at https:// doi.org/10.1007/s40744-021-00314-y.

A. Westerlund · H. Beydogan

Global Database Studies, IQVIA, Stockholm, Sweden

A. M. T. Kejs

Global Database Studies, IQVIA, Copenhagen,

Denmark

K. Gairy (ه)

GlaxoSmithKline, Value Evidence and Outcomes,

Brentford, Middlesex, UK

e-mail: kerry.x.gairy@gsk.com

Present Address:

H. Beydogan

The National Board of Health and Welfare,

Stockholm, Sweden
pSS during follow-up was explored via year-onyear prevalence of and time-to-first extraglandular manifestation. Employment status and retirement rates were used to investigate the impact of pSS on productivity.

Results: A total of 8884 patients with pSS and 88,233 general population comparators were included. Patients with pSS had significantly higher rates of HRU and higher healthcare costs than matched comparators, including twice as many outpatient visits. Costs were highest in year 1 post index before reducing in years 2 and 3 and stabilizing thereafter. Almost two-thirds of patients received their index diagnosis during an outpatient visit, and $<30 \%$ of diagnoses were from rheumatology departments. Overall, $41 \%$ of patients experienced a healthcare encounter that included a relevant extraglandular manifestation code during follow-up. Patients with pSS had significantly higher odds of early retirement than the general population at 5 years post index.

Conclusions: Patients with pSS experience a high clinical and economic disease burden in Sweden.

\section{PLAIN LANGUAGE SUMMARY}

Primary Sjögren's syndrome (pSS) is an autoimmune disease that typically affects the 
secretory glands that produce tears, saliva, and other secretions. Patients can experience debilitating fatigue and can also develop conditions in other parts of the body, commonly including arthritis and Raynaud's phenomenon. This study investigated the burden and disease course of pSS in Sweden. Swedish national registry data were used to match adult patients diagnosed with pSS, with members of the Swedish general population. The use of healthcare resources and their associated costs were compared. The clinical burden of pSS during patient follow-up was explored via year-on-year occurrence and cumulative incidence of extraglandular symptoms. Employment and retirement rates were used to investigate the effect of pSS on patient productivity. A total of 8884 patients with pSS and 88,233 members of the general population were included in the study. Patients with pSS had higher use of healthcare resources and higher associated costs than members of the general population, including twice as many outpatient visits. Costs were highest in the first year after diagnosis, reducing and stabilizing over the following 7 years. Overall, $41 \%$ of patients had a healthcare encounter that included a relevant extraglandular symptom code during follow-up. Almost two-thirds of patients were diagnosed during an outpatient visit, and $<30 \%$ of diagnoses were from rheumatology departments. Patients with pSS were at a greater risk of early retirement than the general population at 5 years post-diagnosis. These results indicate that patients with pSS experience a high disease burden in Sweden.

Keywords: Burden of illness; Healthcare cost/ productivity; Healthcare resource utilization; Primary Sjögren's syndrome; Treatment pathways

\section{Key Summary Points}

\section{Why carry out this study?}

Currently, limited data are available regarding the impact of primary Sjögren's syndrome (pSS) on productivity and gaps in the literature remain for additional contemporary evidence to describe the burden of pSS from a healthcare perspective over time.

This study aimed to investigate the longterm clinical and economic burden of pSS across the entire Swedish population and to describe the disease course (including the development of extraglandular manifestations) and the impact on productivity.

\section{What was learned from the study?}

This study found that patients with pSS experience a high clinical burden in Sweden, where more than $40 \%$ of patients with pSS had at least one extraglandular manifestation during the $>10$-year follow-up period.

Patients with pSS had higher healthcare resource utilization and costs and had four times greater odds of retiring early than the general population.

While not all patients with pSS experience extraglandular involvement, there exists a high economic burden of disease including a substantial cost of drugs prescribed for symptomatic management but without disease-modifying effect.

\section{DIGITAL FEATURES}

This article is published with digital features, including a summary slide and plain language summary to facilitate understanding of the article. To view digital features for this article 
go to https://doi.org/10.6084/m9.figshare.1446 5556.

\section{INTRODUCTION}

Primary Sjögren's syndrome (pSS) is a heterogenous autoimmune disease characterized by lymphocytic infiltration of salivary and lachrymal glands $[1,2]$. It predominantly affects females [1], with a reported prevalence of between 0.01 and $1.56 \%$ [3-5], and an incidence of between three and six cases per 100,000 people [6-10]. Unlike the secondary form of the disease, pSS occurs in the absence of other rheumatic diseases [11]. It has been estimated that $50 \%$ of patients with pSS experience extraglandular manifestations at diagnosis or during a mean follow-up period of 94 months; most commonly presenting as arthritis/arthralgia, but also as other systemic conditions, including Raynaud's phenomenon, peripheral neuropathy, and some instances of interstitial lung disease [12-14]. More than a quarter of patients with pSS have been reported to develop malignancies, with 2.7-9.8\% developing nonHodgkin's lymphoma [15], and irreversible organ damage has been observed to occur, primarily in the parotid glands and eyes $[16,17]$.

Regardless of organ involvement, patients with pSS experience impaired health-related quality of life to a similar degree as patients with rheumatoid arthritis or systemic lupus erythematosus (SLE) $[18,19]$. In addition to dryness, patients with pSS experience significantly more severe pain and fatigue than the general population, resulting in reduced physical functioning, emotional well-being, social functioning, and cognitive impairment [18]. Fatigue is cited by patients as the most severe symptom and the one they most wish they could change $[20,21]$.

The heterogeneous presentations of patients with pSS can make the disease difficult to diagnose and treat. There are currently no available immunomodulatory therapies that have proven efficacy in targeting the underlying pathway(s) of pSS [22] with neither conventional standard nor biologic disease-modifying antirheumatic drugs demonstrating robust or substantial efficacy in large studies [11, 23-32].
Previous studies have revealed the high costs associated with a pSS diagnosis compared with healthy controls $[33,34]$. Similar findings have been reported for Sjögren's syndrome (SS), with or without the presence of other autoimmune conditions [35]. Patients with pSS have been estimated to have 1.6 times greater all-cause healthcare costs than patients without sicca syndrome, with these costs increasing even further for those with extraglandular manifestations [34]. These economic studies have focused on a follow-up duration of 6-24 months [33-35], and clinical studies do not provide insight to the health system burden over time. Data regarding the impact of pSS on productivity are currently limited to single academic cohorts $[32,36]$. This retrospective study of administrative healthcare data aimed to increase understanding of the burden of illness over the $>10$ years of follow-up, and disease course of pSS at a national level in Sweden amongst patients with pSS and matched pSSfree comparators.

\section{METHODS}

\section{Study Design}

This retrospective cohort study was based on data from national registries in Sweden and consisted of patients diagnosed with pSS in secondary care plus members of the Swedish general population. The primary objective was to describe the characteristics of patients with pSS in Sweden and to investigate the associated economic burden to the healthcare system. Secondary objectives were to describe the clinical burden and employment status of patients with pSS in Sweden.

Data were collected for patients diagnosed with pSS between January 1, 2003 and December 31, 2014 (the inclusion period; Fig. 1). The index date was considered the date of diagnosis and patients were required to have 24 months of pre-index data. In addition, a 24-month preinclusion period (January 1, 2001 to December $31,2002)$ was defined to apply the exclusion criteria and ensure that there were no prior diagnosis codes for pSS. The follow-up period 


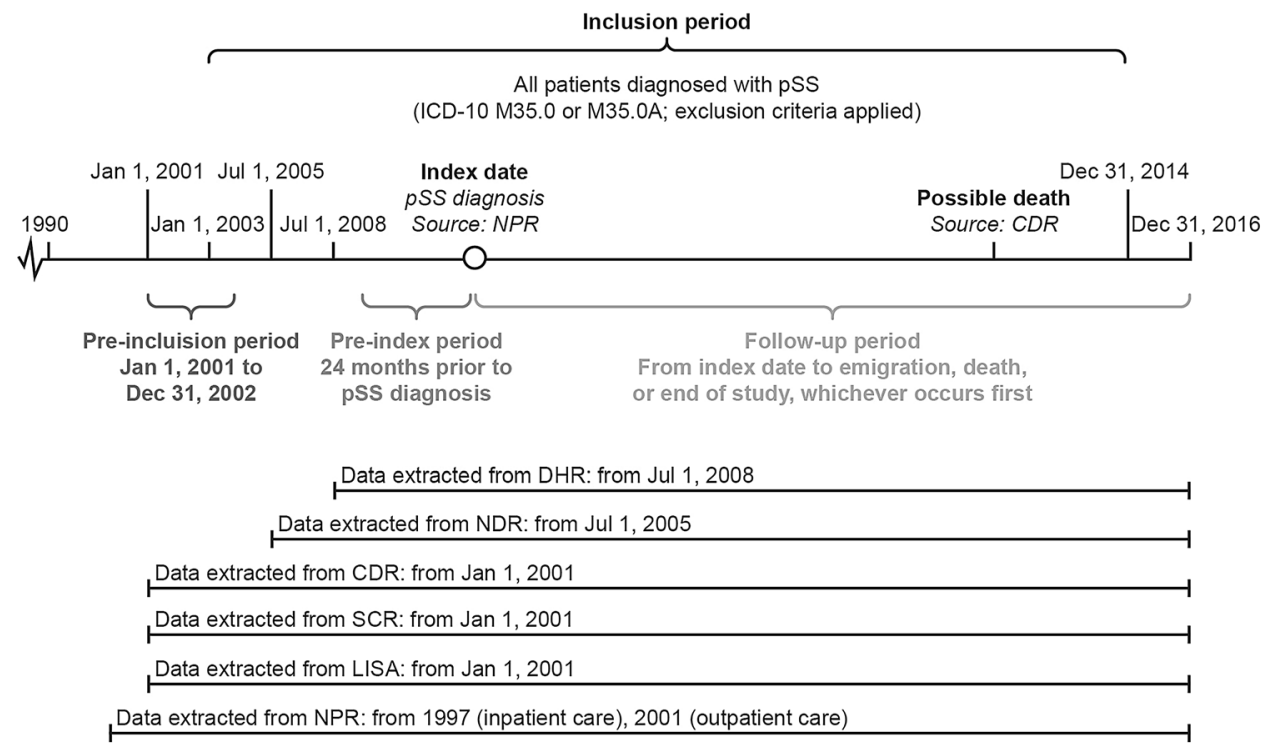

Fig. 1 Study design. $C D R$ Cause of Death Register, $D H R$ Dental Health Register, ICD-10 International Classification of Diseases, Tenth Revision, LISA longitudinal integration database for health insurance and labor market

lasted from the index date until emigration, death, or end of study (December 31, 2016), whichever occurred first. Patients with pSS were identified through the National Patient Register (NPR), with diagnoses based on the International Classification of Diseases, Tenth Revision (ICD-10) codes (Swedish version). Further patient data were extracted from the following national registers: National Prescribed Drug Register (NDR), Cause of Death Register (CDR), Swedish Cancer Registry (SCR), Dental Health Register (DHR), the longitudinal integration database for health insurance and labor market studies (LISA), and the Total Population Register (TPR).

For the general population cohort, each patient with pSS was matched with up to ten comparators on gender, age, and county of residence at index using data from the TPR. Exclusion criteria were not applied to the general population cohort. Patients included in the general population cohort who were diagnosed with pSS during the study period were censored. studies, NDR National Prescribed Drug Register, NPR National Patient Register, $p S S$ primary Sjögren's syndrome, $S C R$ Swedish Cancer Registry

\section{Patients}

Eligible patients were at least 18 years of age at the index date with either a confirmed diagnosis of ICD-10 M35.0 sicca syndrome (Sjögren's; defined as an NPR record of M35.0 for at least two outpatient visits or at least one inpatient stay), or at least one record of ICD-10 M35.0A pSS during the inclusion period. Exclusion criteria included a diagnosis of ICD-10 M05/M06 RA, ICD-10 M32 SLE, or ICD-10 M34 systemic sclerosis up to 6 months after the index date or during the pre-index period, or of ICD-10 M35.0 sicca syndrome (Sjögren's) during the pre-inclusion period; a record of ICD-10 M35.0B secondary SS at least once during the pre-index period; a recorded migration event in the preindex period; and no record within TPR.

Diagnosis codes related to extraglandular manifestations were classified to the following domains based on the European League Against Rheumatism SS disease activity index (ESSDAI) [37]: constitutional, lymphadenopathy and lymphoma, articular, cutaneous, pulmonary, renal, muscular, peripheral nervous system, central nervous system, hematological, and 
biological, and identified by ICD-10 codes. Diagnoses in the ESSDAI glandular domain were similarly identified and reported.

Prevalence-based analyses on cost burden and healthcare resource utilization (HRU) included all patients with pSS with any index date who were in the cohort as of December 31, 2014. Incidence-based analyses were restricted to patients with pSS with an index date in 2009 .

\section{Economic Burden of pSS}

Diagnosis-related group codes were used to estimate the cost per hospitalization, outpatient visit, and medical procedure reported in the NPR. All costs were adjusted to 2016 using the annual healthcare component of the Swedish Consumer Price Index. Descriptions of cost categories are detailed in the Supplementary Methods.

Mean (standard deviation [SD]) and median (interquartile range [IQR]) costs were calculated for patients with pSS and the general population comparators. Since costs were highly skewed, median (IQR) costs are presented. Wilcoxon signed-rank tests were used for statistical comparisons of per-patient median total cost burden between cohorts. For the incidencebased analyses, analysis of covariance (ANCOVA) was used to model total aggregate per-patient costs during follow-up, adjusted for age, gender, county of residence, and comorbidities at index.

\section{Healthcare Resource Utilization}

HRU was reported in terms of hospitalizations, outpatient visits, dental visits, and dental procedures. Mean (SD) and median (IQR) numbers of these healthcare interactions, and length of hospital stays were recorded. Prevalence-based analyses were used to compare HRU between cohorts. A Chi-square test was used for statistical comparisons of number of hospitalizations. $T$ test and Wilcoxon signed-rank test were used for statistical comparisons of mean and median per-patient HRU between cohorts, respectively.

\section{Clinical Burden Over Time}

The excess mortality risk associated with pSS compared with the general population was assessed using Cox proportional hazards regression stratified on matched sets by the level of comorbidity burden. Low comorbidity burden was defined as a low Charlson Comorbidity Index (CCI) score (absence of Charlson comorbidity or a score of 0 or 1 ) during the pre-index period [38]. High comorbidity burden was defined as high CCI score $(\geq 2)$ during the preindex period. Descriptions of the disease manifestation analyses presented in the Supplementary Tables 1 and 2 are included in the Supplementary Methods.

\section{Employment and Retirement Status Over Time}

The distribution of unemployed and retired patients with pSS was described at index and at 1,5 , and 10 years after index. Conditional logistic regression was used to estimate the excess risks of unemployment and early retirement, respectively, at 5 years post index associated with pSS as compared with the general population. Descriptions of the odds ratio (OR) of unemployment and retirement analyses presented in Supplementary Table 3 is included in the Supplementary Methods.

\section{Ethical Considerations}

A copy of the study protocol and all protocol amendments (as needed) were submitted to the Ethical Review Board (ERB) in Stockholm for written approval. ERB approval was obtained before the data-extraction process was initiated. Individual informed consent was not required by Swedish law and no personally identifiable data were extracted. The study population was identified in the NPR by the National Board of Health and Welfare and in the TPR by Statistics Sweden. Personal identification numbers unique to each Swedish citizen or person registered in Sweden were used to link patient data between registers. Pseudonymized patient-level data were subsequently combined into one 
database and delivered to IQVIA for final creation of the study-specific database.

\section{RESULTS}

\section{Study Population}

A total of 8884 patients with pSS and 88,233 general population comparators were included in the study. Both cohorts had a mean age at index of 63 years and were 90\% female. Preindex characteristics and demographics for patients with pSS and the general population comparators are shown in Table 1 . Absence of comorbidities was greater in the general population cohort compared with the pSS cohort (84 vs. $67 \%$, respectively). Amongst CCI comorbidities, patients with pSS compared with the general population had higher relative frequencies of myocardial infarction (3.5 vs. $1.6 \%)$, congestive heart failure (3.7 vs. $1.9 \%)$, chronic pulmonary disease (8.6 vs. $2.7 \%)$, rheumatologic disease (5.7 vs. $1.7 \%)$, mild liver disease ( 2.3 vs. $0.4 \%$ ), and malignancies ( 6.2 vs. $4.0 \%$ ). Overall, $3.2 \%$ and $23.6 \%$ of patients with pSS had at least one diagnosis during the preindex period within the glandular or extraglandular domain, respectively; the most common extraglandular diagnoses were in the articular $(8.6 \%)$ and central nervous system $(4.0 \%)$ domains (Table 1$)$. In the pre-index period, patients with pSS also had a higher prevalence of malaise/fatigue and/or pain diagnosis codes compared with general population comparators (Table 1).

For the majority of the pSS cohort, the index diagnosis was recorded during an outpatient visit ( $n=5784$ [65.1\%]; inpatient visit: $n=3100$ [34.9\%]). The proportion of index diagnoses received from rheumatology departments $(n=2441$ [27.5\%]) was higher than from ophthalmology $(n=1819$ [20.5\%]) and internal medicine $(n=1818 \quad[20.5 \%])$ departments. Additionally, almost one-third of index diagnoses occurred within 62 other medical departments $(n=2806$ [31.6\%]), including surgical $(n=517 \quad[5.8 \%])$, orthopedic $(n=478$ $[5.4 \%])$, or ear, nose, and throat $(n=371$ [4.2\%]) departments.

\section{Prevalent Cohort: Economic Burden of pSS and HRU in 2015}

In 2015, patients with pSS in the prevalent cohort had significantly higher total costs and levels of HRU than the general population comparators (Table 2). Median per-patient drug costs for the pSS cohort were 3.3-fold higher than those of the general population. When broken down into drug categories of interest (identified based on treatment guidelines for pSS) $[31,39,40]$, median costs of drugs providing symptomatic relief of dryness (e.g., eye drops of cyclosporin and pilocarpine) were higher in the pSS cohort than for the general population (Table 2). Fewer than 30\% of patients in the pSS cohort were prescribed drugs in each of the other categories of interest and median costs were therefore low and not different between pSS and general population cohorts. Patients with pSS had a higher median rate of outpatient visits, dental visits, and dental procedures than the general population in 2015 (Table 2). However, $76 \%$ of patients with pSS and $85 \%$ of general population comparators were not hospitalized in 2015 .

\section{Incident Cohort: Economic Burden of pSS Over Time}

Although the mean follow-up time was similar between cohorts, the total costs to the healthcare system and the cost of hospitalizations during the follow-up period for patients diagnosed with pSS in 2009 (incident cohort) were more than double those of the general population comparators (Table 3). Hospitalizations represented most of the costs each year post index. The highest total and hospitalization costs were observed in year 1 before reducing in years 2 and 3, and remaining relatively stable thereafter. Patients with pSS had significantly higher total aggregate per-patient costs over the follow-up period when compared with the general population comparators $(F=26.50$; $p<0.0001$ ) according to adjusted ANCOVA analysis. The median costs of all drugs and drugs of interest were higher in the pSS cohort compared with the general population cohort 
Table 1 Pre-index demographics and clinical characteristics among patients with pSS and general population comparators in the 2-year pre-index period

\begin{tabular}{|c|c|c|}
\hline Characteristic $^{\mathrm{a}}$ & $\begin{array}{l}\text { Patients with pSS } \\
(N=8884)^{\mathrm{b}}\end{array}$ & $\begin{array}{l}\text { General population comparators } \\
(N=\mathbf{8 8 , 2 3 3})\end{array}$ \\
\hline \multicolumn{3}{|l|}{ Age, years } \\
\hline Mean (SD) & $62.8(15.0)$ & $62.9(14.9)$ \\
\hline Median (IQR) & $63.9(53.7-73.8)$ & $64.0(53.8-73.8)$ \\
\hline Range & $(18.0-99.1)$ & $(18.0-99.7)$ \\
\hline \multicolumn{3}{|l|}{ Gender, $n(\%)$} \\
\hline Female & $8033(90.4)$ & $79,776(90.4)$ \\
\hline CCI score, mean $(\mathrm{SD})^{\mathrm{c}}$ & $1.5(1.5)$ & $1.6(1.7)$ \\
\hline \multicolumn{3}{|l|}{ CCI score, $n(\%)^{\mathrm{c}}$} \\
\hline 0 & $631(7.1)$ & $3584(4.1)$ \\
\hline 1 & $1055(11.9)$ & $3833(4.3)$ \\
\hline 2 & $833(9.4)$ & $4847(5.5)$ \\
\hline 3 & $217(2.4)$ & $820(0.9)$ \\
\hline $4+$ & $189(2.1)$ & $1038(1.2)$ \\
\hline Absence of CC & $5959(67.1)$ & $74,111(84.0)$ \\
\hline $\begin{array}{l}\geq 1 \text { diagnosis within glandular domain, }{ }^{\mathrm{d}} \\
n(\%)\end{array}$ & $283(3.2)$ & - \\
\hline Lacrimal swelling & $33(0.4)$ & - \\
\hline Submandibular swelling & $22(0.2)$ & - \\
\hline Parotid swelling & $233(2.6)$ & - \\
\hline $\begin{array}{l}\geq 1 \text { diagnosis within extraglandular domain, }{ }^{\mathrm{d}} \\
n(\%)\end{array}$ & $2098(23.6)$ & - \\
\hline Constitutional domain & $146(1.6)$ & - \\
\hline Lymphadenopathy domain & $136(1.5)$ & - \\
\hline Articular domain & $764(8.6)$ & - \\
\hline Cutaneous domain & $164(1.8)$ & - \\
\hline Pulmonary domain & $89(1.0)$ & - \\
\hline Renal domain & $170(1.9)$ & - \\
\hline Muscular domain & $24(0.3)$ & - \\
\hline Peripheral nervous system domain & $200(2.3)$ & - \\
\hline Central nervous system domain & $357(4.0)$ & - \\
\hline Hematological domain & $296(3.3)$ & - \\
\hline Biological domain & $42(0.5)$ & - \\
\hline
\end{tabular}


Table 1 continued

\begin{tabular}{lll}
\hline Characteristic $^{\mathbf{a}}$ & $\begin{array}{l}\text { Patients with pSS } \\
(\boldsymbol{N}=\mathbf{8 8 8 4})^{\mathbf{b}}\end{array}$ & $\begin{array}{l}\text { General population comparators } \\
(\boldsymbol{N}=\mathbf{8 8 , 2 3 3})\end{array}$ \\
\hline Malaise/fatigue, $n$ (\%) & $104(1.2)$ & $274(0.3)$ \\
Pain, $n$ (\%) & $1127(12.7)$ & $2988(3.4)$ \\
Malaise/fatigue or pain, $n(\%)$ & $1207(13.6)$ & $3225(3.7)$ \\
\hline
\end{tabular}

$C C$ complication or comorbidity, CCI Charlson Comorbidity Index, IQR interquartile range, $p S S$ primary Sjögren's syndrome, $S D$ standard deviation

a All characteristics assessed in the 2-year pre-index period (excluding the index date), unless otherwise indicated

b Percentages based on the total number of patients with pSS (any index date; $N=8884$ )

c The CCI score was calculated for patients with at least one Charlson comorbidity present

${ }^{\mathrm{d}}$ Data for the general population comparators are not available

(Table 3). Additionally, the median costs of drugs for symptomatic relief were higher than the costs of all other drug categories of interest (including antimalarials, corticosteroids, and immunosuppressants) both in year 1 and in total in patients with pSS.

\section{Clinical Burden Over Time}

Overall, $40.7 \%$ of patients with pSS had at least one recorded extraglandular domain manifestation during follow-up (Table S1). The proportion of patients with a recorded extraglandular manifestation decreased yearon-year throughout the entire follow-up period, with 0 events reported in year 10 . At 5 years post index, the proportion of patients that had received a diagnosis of any extraglandular manifestation was 0.314; the articular (0.098), hematological (0.069), and central nervous system (0.064) domains had the highest cumulative incidence (Table S2).

Among those with low comorbidity burden, patients with pSS had a small (12\%) but significantly greater risk of mortality than the general population comparators (hazard ratio [HR; 95\% confidence interval, CI]: 1.12 [1.05-1.19]) (Table 4). In those with a high comorbidity burden, no excess risk of mortality was observed among patients with pSS compared with the general population comparators (HR [95\% CI]: $0.92[0.84-1.00])$.

\section{Impact of pSS on Productivity}

At index, $11 \%$ of patients with pSS who had not already taken normal retirement were unemployed; this proportion declined over the following decade but remained at least double that of the general population (Fig. 2). The OR of unemployment within the pSS cohort was 0.91 (95\% CI 0.61-1.34) compared with the general population at 5 years after index (Table S3). At index, $23.4 \%$ of patients with pSS and $12.1 \%$ of the general population had taken early retirement, while $46.4 \%$ and $47.3 \%$ had retired at or after the retirement age in Sweden (65 years of age [41]), respectively. Over the following decade, rates of early retirement were much greater in the pSS cohort than the general population, with $51.6 \%$ of patients with pSS retiring early at 10 years post index versus $1.8 \%$ in the general population. The OR of early retirement in the pSS cohort was 4.06 (95\% CI 3.24-5.09) compared with the general population at 5 years after index (Table S3).

\section{DISCUSSION}

This retrospective study investigated the burden of pSS on the Swedish healthcare system using administrative healthcare data. It was found that patients with pSS have greater per-person healthcare costs and HRU than the general population and are at a greater risk of early retirement. Although most patients in both 
Table 2 Economic burden of pSS to the Swedish healthcare system and HRU in 2015 (prevalent cohort)

\begin{tabular}{|c|c|c|c|}
\hline & \multirow{2}{*}{$\begin{array}{l}\text { Patients with pSS and } \geq 1 \\
\text { dispensed prescriptions, } n \text { (\%) }\end{array}$} & \multicolumn{2}{|c|}{ Per-patient median (IQR) cost, SEK } \\
\hline & & $\begin{array}{l}\text { Patients with pSS } \\
(N=7396)\end{array}$ & $\begin{array}{l}\text { General population } \\
(N=76,624)\end{array}$ \\
\hline Total cost & - & $\begin{array}{l}23,735 \\
\quad(9907-58,180)\end{array}$ & $8128(2822-24,540)^{\mathrm{c}}$ \\
\hline Hospitalization costs & - & $0(0-0)$ & $0(0-0)$ \\
\hline Outpatient visit costs & - & $\begin{array}{l}7961 \\
\quad(2485-20,177)\end{array}$ & $2209(0-8145)$ \\
\hline Dental visit costs & - & $1793(0-3894)$ & $1190(0-2804)$ \\
\hline Drug costs & - & $\begin{array}{l}5384 \\
\quad(2142-11,880)\end{array}$ & $1630(400-4770)$ \\
\hline Drugs of interest ${ }^{\mathrm{d}}$ & - & $1082(276-2685)$ & $57(0-475)$ \\
\hline Biologic therapy $^{\mathrm{e}}$ & $15(0.2)$ & $0(0-0)$ & $0(0-0)$ \\
\hline $\begin{array}{l}\text { Antimalarials, corticosteroids, and } \\
\text { immunosuppressants }^{f}\end{array}$ & $2135(28.9)$ & $0(0-97)$ & $0(0-0)$ \\
\hline Symptomatic relief of dryness ${ }^{\mathrm{g}}$ & $4386(59.3)$ & $165(0-932)$ & $0(0-0)$ \\
\hline Topical fluoride and chlorhexidine & $1550(21.0)$ & $0(0-0)$ & $0(0-0)$ \\
\hline
\end{tabular}

\section{Number of healthcare interactions}

Hospitalizations, $n(\%)$

0

1

2

$\geq 3$

Hospitalizations

Mean (SD)

Median (IQR)

Length of stay, days

Mean (SD)

Median (IQR)

Outpatient visits

Mean (SD)

Median (IQR)

Dental visits

Mean (SD)

Median (IQR)
$5652(76.4)$

1006 (13.6)

$362(4.9)$

$376(5.1)$

$0.5(1.2)$

$0.0(0.0-0.0)$

$2.6(9.4)$

$0.0(0.0-0.0)$

4.4 (6.3)

$3.0(1.0-6.0)$

$2.3(2.3)$

$2.0(0.0-3.0)$
65,034 (84.9 $)^{\mathrm{c}}$

$6700(8.7)^{\mathrm{c}}$

$2558(3.3)^{\mathrm{c}}$

$2332(3.0)^{\mathrm{c}}$

$0.3(0.9)^{c}$

$0.0(0.0-0.0)$

$0.0(0.0-0.0)$

$1.6(7.5)^{\mathrm{c}}$

$2.0(4.1)^{\mathrm{c}}$

$1.0(0.0-3.0)$

$1.6(1.9)^{\mathrm{c}}$

$1.0(0.0-2.0)$ 
Table 2 continued

\section{Number of healthcare interactions}

Dental procedures
Mean (SD)
$3.8(4.0)$
$2.8(3.3)^{\mathrm{c}}$
Median (IQR)
$3.0(0.0-6.0)$
$2.0(0.0-4.0)$
$H R U$ healthcare resource utilization, $I Q R$ interquartile range, $p S S$ primary Sjögren's syndrome, $S D$ standard deviation, $S E K$ Swedish Krona
${ }^{a}$ Based on patients/individuals (any index date) in the cohort on December 31, 2014
b HRU reported as number of healthcare interactions unless otherwise indicated
${ }^{c} p<0.0001$
d Based on the European League Against Rheumatism (EULAR) and Sjögren's Foundation guidelines, and supplemented with the AECG guidelines for procedures of interest [31, 39, 40]
e Rituximab, belimumab, abatacept, tocilizumab
f Hydroxychloroquine, methotrexate, corticosteroids, azathioprine, cyclosporine, leflunomide, sulfasalazine
g Artificial tears (e.g., sodium hyaluronate, hypromellose), cyclosporin (ophthalmic), pilocarpine, cevimeline, topical autologous serum, methylcellulose inserts, minocycline (oral), doxycycline (oral), artificial saliva

cohorts had no hospitalizations in 2015, the cost per patient per hospitalization was greater for patients with pSS. Similarly, patients with pSS had greater drug costs relating to symptomatic relief of dryness than general population comparators. Patients with pSS also had greater drug costs for symptomatic relief of dryness compared with the costs for other drug categories of interest both in year 1 and in total.

These results are consistent with previous studies into the socioeconomic burden of pSS on patients. Mandl et al. investigated rates of work disability in Sweden and found that patients with pSS have more than double the risk of sick leave or work disability 2 years after diagnosis when compared with the general population [36]. Higher levels of HRU amongst patients with pSS than the general population have also been reported [42]. A previous study in a Dutch SS population found a significantly lower employment rate compared with the general population [32]; however, we found that patients with pSS have similar odds of employment to the general population in Sweden. This suggests that the impact of pSS on work productivity in Sweden may be a result of early retirement rather than unemployment.

Patients with pSS most commonly received their index diagnosis through rheumatology, ophthalmology, and internal medicine departments. However, index diagnoses were reported for some patients in a wide range of different departments. These data indicate that the selection criteria were sensitive, despite attempts to improve selectivity. These findings also suggest that the first record of pSS in the secondary care system is the first encounter of a patient already diagnosed in primary care. Therefore, the variety of medical specialties reflects the heterogeneity of manifestations and comorbidities requiring attention in this population.

Patients with pSS experience an increase in extraglandular manifestations after diagnosis [43], highlighting the high clinical burden of the disease. In this study, the 5-year cumulative incidence of extraglandular manifestations (0.314) suggests that patients with pSS receive a diagnosis of manifestations early. This is supported by previous studies that show high healthcare costs shortly after diagnosis [33-35]. These findings indicate that few patients receive treatment for pSS within specialized care in Sweden, highlighting an unmet clinical need. In this study, patients had the highest healthcare costs in the first year after diagnosis, with these costs decreasing and stabilizing over the following 7 years. This may be due to intensive 
Table 3 Economic burden of pSS to the healthcare system during the follow-up period among patients with pSS diagnosed in 2009 (incident cohort), by cost categories

\begin{tabular}{|c|c|c|c|}
\hline & \multicolumn{3}{|c|}{ Per-patient median (IQR), SEK } \\
\hline & \multicolumn{2}{|c|}{ Patients with pSS $(N=692)$} & \multirow{2}{*}{$\begin{array}{l}\text { General population } \\
(N=\mathbf{6 8 5 9}) \\
6.9(1.7)\end{array}$} \\
\hline Mean time of follow-up, years (SD) & $6.6(2.0)$ & & \\
\hline & Year 1 & Total & Total \\
\hline Total cost & $\begin{array}{l}38,781 \\
\quad(17,290-80,161)\end{array}$ & $\begin{array}{l}245,556 \\
\quad(136,586-420,567)\end{array}$ & $99,408(42,648-216,084)^{a}$ \\
\hline Hospitalizations & $13,674(0-48,026)$ & $\begin{array}{l}86,003 \\
\quad(17,430-204,149)\end{array}$ & $23,379(0-102,373)$ \\
\hline Outpatient visits & $\begin{array}{l}8101 \\
\quad(3052-16,166)\end{array}$ & $\begin{array}{l}61,112 \\
\quad(29,967-110,956)\end{array}$ & $21,795(7049-49,741)$ \\
\hline Dental visits & $937(0-2408)$ & $\begin{array}{l}14,872 \\
\quad(4707-30,055)\end{array}$ & $11,605(3299-23,018)$ \\
\hline Drugs (all) & $\begin{array}{l}6550 \\
\quad(2802-14,090)\end{array}$ & $\begin{array}{l}41,662 \\
\quad(19,453-86,637)\end{array}$ & $13,702(4156-35,902)$ \\
\hline Drugs of interest ${ }^{\mathrm{b}}$ & $1406(444-2961)$ & $9290(2917-17,948)$ & $788(125-3413)$ \\
\hline Biologic therapy ${ }^{c}$ & $0(0-0)$ & $0(0-0)$ & $0(0-0)$ \\
\hline $\begin{array}{l}\text { Antimalarials, corticosteroids, and } \\
\text { immunosuppressants }{ }^{\mathrm{d}}\end{array}$ & $0(0-111)$ & $70(0-783)$ & $0(0-59)$ \\
\hline Symptomatic relief of dryness ${ }^{\mathrm{e}}$ & $400(0-1239)$ & $1946(352-7672)$ & $0(0-120)$ \\
\hline Topical fluoride and chlorhexidine & $0(0-24)$ & $0(0-335)$ & $0(0-0)$ \\
\hline
\end{tabular}

$I Q R$ interquartile range, $p S S$ primary Sjögren's syndrome, $S D$ standard deviation, $S E K$ Swedish Krona

${ }^{\mathrm{a}} p<0.0001$

b Based on the European League Against Rheumatism (EULAR) and Sjögren's Foundation guidelines, and supplemented with the AECG guidelines for procedures of interest $[31,39,40]$

c Rituximab, belimumab, abatacept, tocilizumab

${ }^{\mathrm{d}}$ Hydroxychloroquine, methotrexate, corticosteroids, azathioprine, cyclosporine, leflunomide, sulfasalazine

e Artificial tears (e.g., sodium hyaluronate, hypromellose), cyclosporin (ophthalmic), pilocarpine, cevimeline, topical autologous serum, methylcellulose inserts, minocycline (oral), doxycycline (oral), artificial saliva

treatment efforts shortly after diagnosis, followed by stabilized treatment regimens that do not appear to reduce the disease burden, as healthcare costs do not decrease year-on-year. Decreasing healthcare costs over time could be expected to occur following the control of a patient's disease activity with adequate therapies. However, due to the lack of effective licensed treatments for pSS, it is more likely that patients simply learn to endure their symptoms.
The high levels of healthcare costs after diagnosis are consistent with those seen in a previous US study, which reported that SS is associated with $40 \%$ increased all-cause healthcare costs in the first year after diagnosis compared with the year before [35].

This study is not without limitations. Patients had a higher mean age than would be typically expected of a pSS population [42], suggesting that there may be some 
Table 4 Median survival and risk of death among patients with pSS compared with the general population among those with low and high comorbidity burden ${ }^{\mathrm{a}}$

\begin{tabular}{lllll}
\hline & At risk & Events & Median survival, years (95\% CI) & HR (95\% CI) \\
\hline Low comorbidity burden $^{\mathbf{c}}$ & & & & \\
General population comparators & 81,652 & 11,515 & NA & 1 (reference) \\
$\begin{array}{l}\text { Patients with pSS } \\
\text { High comorbidity burden }\end{array}$ & 7677 & 1173 & NA & $1.12(1.05-1.19)$ \\
General population comparators & 6581 & 3206 & $7.20(6.92-7.53)$ & 1 (reference) \\
Patients with pSS & 1207 & 562 & $8.20(7.41-8.80)$ & $0.92(0.84-1.00)$ \\
\hline
\end{tabular}

$C C I$ Charlson Comorbidity Index, $C I$ confidence interval, $H R$ hazard ratio, IQR interquartile range, $p S S$ primary Sjögren's syndrome

a Based on patients/individuals with any index date ( $N$, patients with $\mathrm{pSS}=8884 ; N$, general population comparators $=88,233$ )

${ }^{\mathrm{b}}$ By design of matching, HR adjusted for age, gender, and county of residence at index

${ }^{c}$ Low comorbidity burden defined as low CCI score (absence of Charlson comorbidity or CCI score 0-1) during the preindex period

${ }^{\mathrm{d}}$ High comorbidity burden defined as high CCI score $(\geq 2)$ during the pre-index period

false-positive individuals within the population who do not truly have pSS. As with many retrospective cohort studies, patient diagnoses were based on codes within a national administrative register, meaning that there is a risk that some patients were misclassified or had other diagnoses than those described here. However, this may instead be a result of patients with pSS entering secondary care later in the disease pathway, which would mean that the burden of the disease is underestimated in this study. Similarly, the study population may be more severe than the general pSS population, as patients treated within a primary care setting were not included and would be likely to have less severe symptoms than those treated in secondary care. In addition, although relevant codes do exist for many key pSS manifestations (such as peripheral neuropathy and pulmonary interstitial fibrosis), some symptoms of pSS (including vaginal dryness) could not be analyzed, as there is no relevant ICD-10 code. In the present study, only $\sim 40 \%$ of the cohort had manifestations captured by the ESSDAI domains. While, in part, this may be due to miscoding or a lack of specific diagnosis codes, it likely reflects that the most bothersome aspects of pSS (i.e., pain, dryness, and fatigue) are not well captured in secondary data sources. This is supported by the low pre-index prevalence of malaise/fatigue and/or pain in patients with pSS. Despite being reported as the most bothersome symptom of pSS [20,21], only $1.2 \%$ of patients with pSS had a recorded diagnosis for malaise/fatigue in the pre-index period. However, the diagnosis code for malaise/fatigue had limited utility since further clinical data and laboratory data are not captured in the Swedish national patient register. Nonetheless, the data in this study reflect the total cost of this population to the secondary healthcare system, and the overall burden will incorporate all diseaserelated burdens regardless of whether a specific symptom can be identified. Furthermore, not all procedures may have been coded within the registries used. In particular, manifestations for which there are no specific reimbursable treatments, such as fatigue or glandular swelling, may not have been coded. Fatigue and depression in patients with pSS can contribute to a greater individual and societal burden than the classical ocular and oral symptoms of the disease [42], and therefore are likely to increase further the clinical and healthcare burden in patients with pSS. Given that some manifestations of pSS could also be captured by the CCI, 

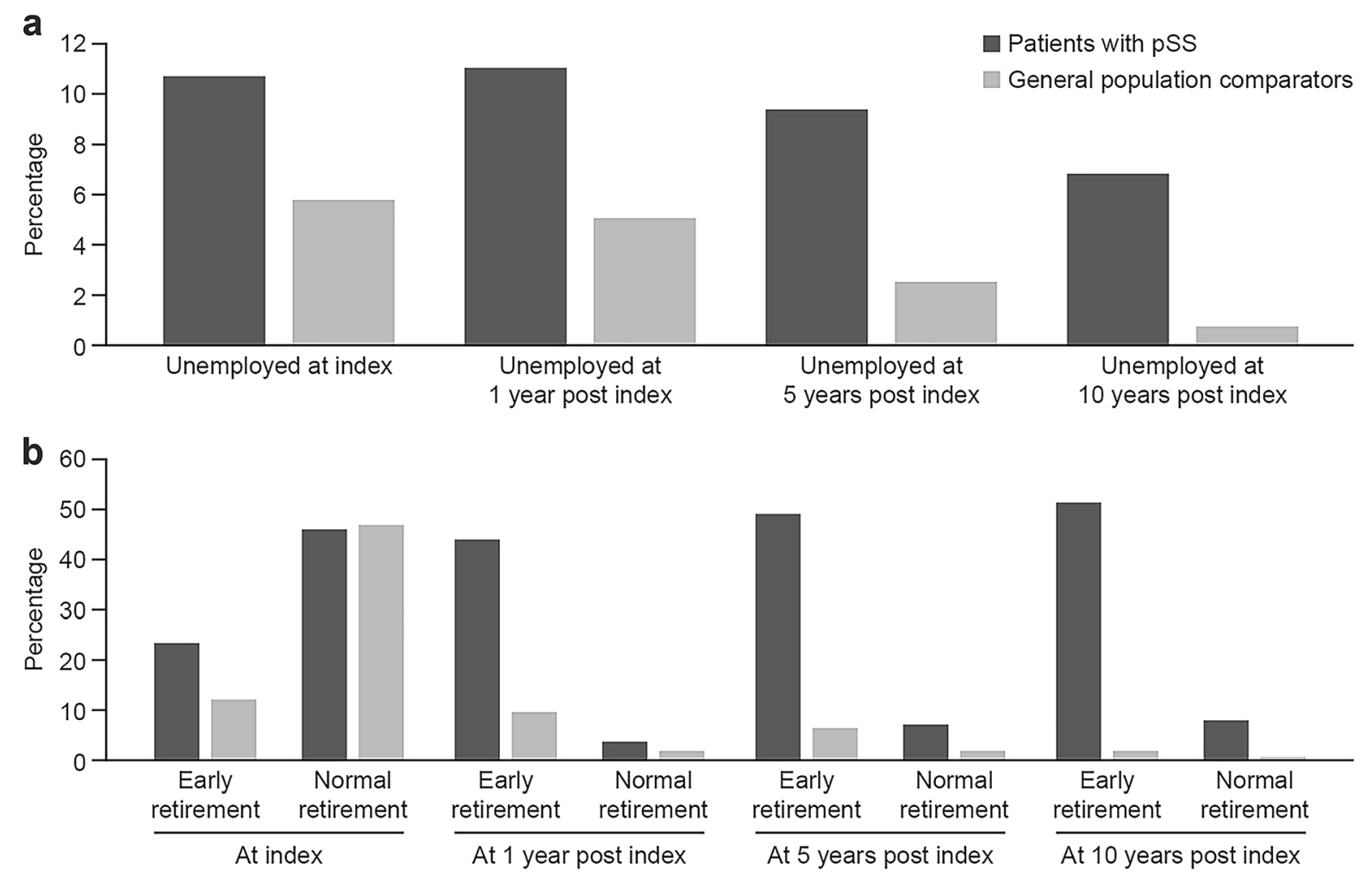

Fig. 2 Employment (a) and retirement (b) status at and over time after index among patients with pSS and general population comparators. $p S S$ primary Sjögren's syndrome. Unless otherwise indicated, percentages are based on the cohort at risk at each time point for assessment (i.e.,

general population cohorts were not matched based on this characteristic. This could be another limitation of the analysis, as the pSS cohort has a greater degree of comorbidities. However, since it is unknown whether these comorbidities are related to this diagnosis, the objective of this study was to capture all the excess burden in patients with pSS.

Further research is needed to validate the ICD-10 codes used to identify pSS and to more clearly identify which aspects of HRU might be influenced by future treatments options and unmet medical needs. In the Swedish setting, this could be achieved by combining these national register data with patient-level data reported in electronic medical records, as well as through linkage to specific pSS clinical registries. Also, it would be of interest to compare use of topical drugs in patients with pSS with excluding from the denominator the number of patients who had retired [old-age pension] or had been censored due to death, emigration, or end of study). Normal retirement was defined as having drawn an old-age pension in the calendar year of turning 65 years of age or older

patients without pSS but experiencing sicca symptoms, in order to establish the contribution of such treatments to the higher HRU in patients with pSS. This comparison could not be reliably made in the current study; it is likely that ICD-10 codes such as 'dryness of mouth' were underreported, since only $3.8 \%$ of the pSS cohort had a record of this diagnosis code in the 2-year pre-index period (data not shown), despite this being a cardinal symptom.

\section{CONCLUSIONS}

This retrospective study reveals the substantial economic and clinical burden associated with pSS in Sweden. Novel treatments that can alleviate the burden associated with pSS would 
likely be welcomed by patients and healthcare professionals alike.

\section{ACKNOWLEDGEMENTS}

We thank all the patients and their families, and the clinicians, who provided data for this study.

Funding. This study was funded by GlaxoSmithKline, Brentford, UK (study number 206587 [HO-15-16079]). The journal's Rapid Service Fee was also funded by GlaxoSmithKline, Brentford, UK.

Medical Writing, Editorial, and Other Assistance. Medical writing support was provided by Sam Halliwell, PhD, and Liam Campbell, PhD, of Fishawack Indicia Ltd, UK, and was funded by GlaxoSmithKline. The authors would like to acknowledge Chi Truong, $\mathrm{MD}, \mathrm{PhD}$, for her contributions to the review of the ICD-10 codes used in this study. The authors also wish to acknowledge the contribution of Lisen Arnheim Dahlström to the development of the protocol for this study.

Authorship. All named authors meet the International Committee of Medical Journal Editors (ICMJE) criteria for authorship for this article, take responsibility for the integrity of the work as a whole, and have given their approval for this version to be published.

Author Contributions. Kerry Gairy and Anna Westerlund designed the study, Anna Westerlund was also responsible for data acquisition. All authors contributed to the data analysis and interpretation.

Prior Presentation. Patient demographics as well as components of the cost, number of hospitalizations, length of hospital stay, odds of unemployment, and early retirement, all compared to the general population, have been previously presented at the 2019 International Society of Pharmacoeconomics and Outcomes Research conference (Westerlund A, Beydogan H, Kejs AM, Gairy K. PSY10 primary Sjögren's syndrome: a retrospective cohort study of burden of illness in Sweden. Value in Health. 2019;22:S902-S3.).

Disclosures. Kerry Gairy is an employee of GlaxoSmithKline and holds shares in the company. Anna Westerlund and Anne Mette Tranberg Kejs are employees of IQVIA, a service provider that performed this retrospective cohort study on behalf of GlaxoSmithKline. Heval Beydogan was an employee of IQVIA at the time of the study; their current employment address is The National Board of Health and Welfare, Stockholm, Sweden.

Compliance with Ethics Guidelines. A copy of the study protocol was submitted to the Ethical Review Board (ERB) in Stockholm for written approval. ERB approval was obtained before the data-extraction process was initiated. The investigator submitted and, as needed, obtained approval from the ERB for all protocol amendments. Individual informed consent was not required by Swedish law and no personally identifiable data were extracted. The study population was identified in the NPR by the National Board of Health and Welfare and in the TPR by Statistics Sweden. Personal identification numbers unique to each Swedish citizen or person registered in Sweden were used to link patient data between registers. Pseudonymized patient-level data were subsequently combined into one database and delivered to IQVIA for final creation of the study-specific database.

Data Availability. The datasets generated and analyzed during the current study are not publicly available due to extraction from national data registries and for data protection, but are available from the Swedish National Board of Health and Welfare upon request (https://www.socialstyrelsen.se/statistik-ochdata/register/).

Open Access. This article is licensed under a Creative Commons Attribution-NonCommercial 4.0 International License, which permits any non-commercial use, sharing, adaptation, distribution and reproduction in any medium or format, as long as you give appropriate credit to the original author(s) and the source, provide 
a link to the Creative Commons licence, and indicate if changes were made. The images or other third party material in this article are included in the article's Creative Commons licence, unless indicated otherwise in a credit line to the material. If material is not included in the article's Creative Commons licence and your intended use is not permitted by statutory regulation or exceeds the permitted use, you will need to obtain permission directly from the copyright holder. To view a copy of this licence, visit http://creativecommons.org/licenses/by$\mathrm{nc} / 4.0 /$.

\section{REFERENCES}

1. Dumusc A, Bowman SJ. Sjögren syndrome and work disability. J Rheumatol. 2017;44:133-5.

2. Ferro F, Marcucci E, Orlandi M, Baldini C, Bartoloni-Bocci E. One year in review 2017: primary Sjögren's syndrome. Clin Exp Rheumatol. 2017;35: 179-91.

3. Retamozo S, Flores-Chavez A, Consuegra-Fernandez M, Lozano F, Ramos-Casals M, Brito-Zeron P. Cytokines as therapeutic targets in primary Sjögren syndrome. Pharmacol Ther. 2018;184:81-97.

4. Maldini C, Seror R, Fain O, Dhote R, Amoura Z, De Bandt M, et al. Epidemiology of primary Sjögren's syndrome in a French multiracial/multiethnic area. Arthritis Care Res (Hoboken). 2014;66:454-63.

5. Kabasakal Y, Kitapcioglu G, Turk T, Oder G, Durusoy R, Mete N, et al. The prevalence of Sjögren's syndrome in adult women. Scand J Rheumatol. 2006;35:379-83.

6. Pillemer SR, Matteson EL, Jacobsson LT, Martens $\mathrm{PB}$, Melton LJ 3rd, O'Fallon WM, et al. Incidence of physician-diagnosed primary Sjögren syndrome in residents of Olmsted County, Minnesota. Mayo Clin Proc. 2001;76:593-9.

7. Plesivcnik Novljan M, Rozman B, Hocevar A, Grmek $\mathrm{M}$, Kveder $\mathrm{T}$, Tomsic $\mathrm{M}$. Incidence of primary Sjögren's syndrome in Slovenia. Ann Rheum Dis. 2004;63:874-6.

8. Alamanos $\mathrm{Y}$, Tsifetaki N, Voulgari PV, Venetsanopoulou AI, Siozos C, Drosos AA. Epidemiology of primary Sjögren's syndrome in north-west Greece, 1982-2003. Rheumatology (Oxford). 2006;45:187-91.
9. Weng MY, Huang YT, Liu MF, Lu TH. Incidence and mortality of treated primary Sjögren's syndrome in Taiwan: a population-based study. J Rheumatol. 2011;38:706-8.

10. Kvarnstrom M, Ottosson V, Nordmark B, WahrenHerlenius M. Incident cases of primary Sjögren's syndrome during a 5-year period in Stockholm County: a descriptive study of the patients and their characteristics. Scand J Rheumatol. 2015;44: $135-42$.

11. Del Papa N, Vitali C. Management of primary Sjögren's syndrome: recent developments and new classification criteria. Ther Adv Musculoskelet Dis. 2018;10:39-54.

12. Ramos-Casals M, Brito-Zeron $\mathrm{P}$, Perez-De-Lis $\mathrm{M}$, Jimenez I, Blanco MJ, Bove A, et al. Sjögren syndrome or Sjögren disease? The histological and immunological bias caused by the 2002 criteria. Clin Rev Allergy Immunol. 2010;38:178-85.

13. Brito-Zerón P, Acar-Denizli N, Zeher M, Rasmussen A, Li X, Baldini C, et al. THU0681 baseline ESSDAI/ DAS scores in 8061 patients with primary Sjögren syndrome: characterization of Systemic disease. Ann Rheum Dis. 2017;76:464-5.

14. Brito-Zerón P, Acar-Denizli N, Ng WF, Zeher M, Rasmussen A, Mandl T, et al. How immunological profile drives clinical phenotype of primary Sjögren's syndrome at diagnosis: analysis of 10,500 patients (Sjögren Big Data Project). Clin Exp Rheumatol. 2018;112:102-12.

15. Alunno A, Leone MC, Giacomelli R, Gerli R, Carubbi F. Lymphoma and lymphomagenesis in primary Sjögren's syndrome. Front Med (Lausanne). 2018;5:102.

16. Sutcliffe N, Stoll T, Pyke S, Isenberg DA. Functional disability and end organ damage in patients with systemic lupus erythematosus (SLE), SLE and Sjögren's syndrome (SS), and primary SS. J Rheumatol. 1998;25:63-8.

17. Krylova L, Isenberg D. Assessment of patients with primary Sjögren's syndrome-outcome over 10 years using the Sjögren's Syndrome Damage Index. Rheumatology (Oxford). 2010;49:1559-62.

18. Segal B, Bowman SJ, Fox PC, Vivino FB, Murukutla N, Brodscholl J, et al. Primary Sjögren's syndrome: health experiences and predictors of health quality among patients in the United States. Health Qual Life Outcomes. 2009; 7:46.

19. Kotsis K, Voulgari PV, Tsifetaki N, Drosos AA, Carvalho $\mathrm{AF}$, Hyphantis $\mathrm{T}$. Illness perceptions and psychological distress associated with physical health-related quality of life in primary Sjögren's 
syndrome compared to systemic lupus erythematosus and rheumatoid arthritis. Rheumatol Int. 2014;34:1671-81.

20. Bowman SJ. Patient-reported outcomes including fatigue in primary Sjögren's syndrome. Rheum Dis Clin N Am. 2008;34(949-62):ix.

21. Gairy K, Ruark K, Sinclair SM, Brandwood H, Nelsen L. An innovative online qualitative study to explore the symptom experience of patients with primary Sjögren's syndrome. Rheumatol Ther. 2020;7: 601-15.

22. Felten R, Scher F, Sibilia J, Gottenberg JE, Arnaud L. The pipeline of targeted therapies under clinical development for primary Sjögren's syndrome: a systematic review of trials. Autoimmun Rev. 2019; 18:576-82.

23. Radstake TRDJ, van der Heijden EHM, Moret FM, Hillen MR, Lopes AP, Rosenberg T, et al. Clinical efficacy of leflunomide/hydroxychloroquine combination therapy in patients with primary Sjögren's syndrome: results of a placebo-controlled doubleblind randomized clinical trial [abstract]. Arthritis Rheumatol. 2018;70(suppl 10).

24. Devauchelle-Pensec V, Mariette X, Jousse-Joulin S, Berthelot JM, Perdriger A, Puéchal X, et al. Treatment of primary Sjögren syndrome with rituximab: a randomized trial. Ann Intern Med. 2014;160: 233-42.

25. Bowman SJ, Everett CC, O'Dwyer JL, Emery P, Pitzalis C, Ng WF, et al. Randomized controlled trial of rituximab and cost-effectiveness analysis in treating fatigue and oral dryness in primary Sjögren's syndrome. Arthritis Rheumatol. 2017;69: 1440-50.

26. Dass S, Bowman SJ, Vital EM, Ikeda K, Pease CT, Hamburger J, et al. Reduction of fatigue in Sjögren syndrome with rituximab: results of a randomised, double-blind, placebo-controlled pilot study. Ann Rheum Dis. 2008;67:1541-4.

27. Meijer JM, Meiners PM, Vissink A, Spijkervet FK, Abdulahad W, Kamminga N, et al. Effectiveness of rituximab treatment in primary Sjögren's syndrome: a randomized, double-blind, placebo-controlled trial. Arthritis Rheum. 2010;62:960-8.

28. Baer A, Gottenberg JE, St. Clair EW, Sumida T, Takeuchi T, Seror R, et al. OP0039 efficacy and safety of abatacept in active primary Sjögren's syndrome: results of a randomised placebo-controlled phase III trial. Ann Rheum Dis. 2019;78:89-90.

29. Felten R, Devauchelle-Pensec V, Seror R, Duffau P, Saadoun D, Hachulla E, et al. Interleukin 6 receptor inhibition in primary Sjögren syndrome: a multicentre double-blind randomised placebo-controlled trial. Ann Rheum Dis. 2021;80:329-38.

30. Mariette X, Seror R, Quartuccio L, Baron G, Salvin S, Fabris M, et al. Efficacy and safety of belimumab in primary Sjögren's syndrome: results of the BELISS open-label phase II study. Ann Rheum Dis. 2013;74: 526-31.

31. Vivino FB, Carsons SE, Foulks G, Daniels TE, Parke A, Brennan MT, et al. New treatment guidelines for Sjögren's disease. Rheum Dis Clin N Am. 2016;42: 531-51.

32. Meijer JM, Meiners PM, Huddleston Slater JJ, Spijkervet FK, Kallenberg CG, Vissink A, et al. Healthrelated quality of life, employment and disability in patients with Sjögren's syndrome. Rheumatology (Oxford). 2009;48:1077-82.

33. Callaghan R, Prabu A, Allan RB, Clarke AE, Sutcliffe $\mathrm{N}$, Pierre YS, et al. Direct healthcare costs and predictors of costs in patients with primary Sjögren's syndrome. Rheumatology (Oxford). 2007;46: 105-11.

34. Perera S, Ma L, Punwaney R, Ramachandran S. FRI0763-HPR investigating the clinical and cost burden of primary Sjögren's syndrome: a descriptive analysis from a us administrative claims database. J Health Econ Outcomes Res. 2017;5(1506): 2-7.

35. Birt JA, Tan Y, Mozaffarian N. Sjögren's syndrome: managed care data from a large United States population highlight real-world health care burden and lack of treatment options. Clin Exp Rheumatol. 2017;35:98-107.

36. Mandl T, Jørgensen TS, Skougaard M, Olsson P, Kristensen LE. Work disability in newly diagnosed patients with primary Sjögren syndrome. J Rheumatol. 2017;44:209-15.

37. Seror R, Ravaud P, Bowman SJ, Baron G, Tzioufas A, Theander E, et al. EULAR Sjögren's syndrome disease activity index: development of a consensus systemic disease activity index for primary Sjögren's syndrome. Ann Rheum Dis. 2010;69:1103-9.

38. Quan H, Li B, Couris CM, Fushimi K, Graham P, Hider $\mathrm{P}$, et al. Updating and validating the Charlson comorbidity index and score for risk adjustment in hospital discharge abstracts using data from 6 countries. Am J Epidemiol. 2011;173:676-82.

39. Vitali C, Bombardieri S, Jonsson R, Moutsopoulos HM, Alexander EL, Carsons SE, et al. Classification criteria for Sjögren's syndrome: a revised version of the European criteria proposed by the American-European Consensus Group. Ann Rheum Dis. 2002;61:554-8. 
40. Ramos-Casals M, Brito-Zeron P, Bombardieri S, Bootsma H, De Vita S, Dorner T, et al. EULAR recommendations for the management of Sjögren's syndrome with topical and systemic therapies. Ann Rheum Dis. 2020;79:3-18.

41. European Commission. Sweden-Old-age pension. https://www.ec.europa.eu/social/main.jsp?catId= 1130\&langId=en\&intPageId $=4814$. Accessed 6 May 2019.

42. Westhoff G, Dörner T, Zink A. Fatigue and depression predict physician visits and work disability in women with primary Sjögren's syndrome: results from a cohort study. Rheumatology (Oxford). 2012;51:262-9.

43. Ramos-Casals M, Brito-Zeron P, Solans R, Camps MT, Casanovas A, Sopena B, et al. Systemic involvement in primary Sjögren's syndrome evaluated by the EULAR-SS disease activity index: analysis of 921 Spanish patients (GEAS-SS Registry). Rheumatology (Oxford). 2014;53:321-31. 